\title{
UNDERWATER DELOYMENT ROBOTS - FROM AMOEBOT TO ROBOGLIDER
}

\section{I-Ming Chen Hsi-Shang Li Francis M. J. Nickols Xinggang Yan}

\author{
School of Mechanical and Production Engineering \\ Nanyang Technological University \\ Nanyang Ave, Singapore 639798 \\ Email: \{ michen, mhsli, mfnickols, mxgyan \} @ ntu.edu.sg
}

\begin{abstract}
Two types of biologically-inspired underwater robotic systems are introduced for underwater deployment and inspection purpose: 1) Amoebot, a shape-changing robot that mimics the swimming action of microorganism; 2) RoboGlider, an under-actuated 1DOF robotic platform whose locomotion is inspired by the phenomenon of falling leaf or paper. The movement of those underwater systems can be explained using the concept of dynamical systems modeled upon the solid-fluid interactions. Computer simulation of the locomotion and construction of experimental prototypes are investigated and discussed.
\end{abstract}

Keywords: Underwater robot. Underwater deployment. Biomimetic robot. Nonlinear dynamical systems.

\section{INTRODUCTION}

Underwater or undersea deployment of artificial objects is currently performed using divers or conventional submersible vehicles. Because of the hostile and unpredictable underwater environment, autonomous and semi-autonomous underwater vehicles are gaining popularity in performing such tasks. Those vehicles use propellers for propulsion and rudders for controlling the direction of travel. Such kind of propulsion systems not only generate significant amount of noise but also has low efficiency, making the vessels unfriendly to marine ecology and environment and energy-inefficient. Quite contrary in the animal world, marine mammals and microorganisms usually propel in the water through continuous change of the body configurations without resorting to the propellers. Such propulsive principle is developed by creatures naturally and extremely energy efficient.

In this article, we describe a project aiming at developing propeller-less biologically-inspired underwater vehicle systems as an alternative to the existing submersible systems for deployment and inspection purpose. The robotic underwater vehicle systems developed based on the principle of marine life will possess similar advantages over the conventional systems. Two different types of underwater robotic systems are introduced. 1) Amoebot - a Metamorphic Underwater Vehicle (MUV) whose locomotion is inspired by the selfpropulsion of the microorganism, amoebae [1]. The microorganism can propel itself in very low Reynolds number flow through the shape change of its body. Similarly, through the change of the body shape, the MUV can swim silently and has the capability to pass regions with obstacles under the water (Fig.1). 2) RoboGlider - an under-actuated underwater deployment robotic platform whose locomotion is inspired by the phenomena of falling leaf or paper [2]. A leaf or paper, when falling to the ground under the gravitation force, exhibits complex dynamical behaviors because of the lift and drag in solid-fluid interaction. As the shape parameters of the falling object can be modified, the trajectory of the object in the fluid (air or water) can be controlled. The RoboGlider thus travels underwater through the change of its critical dimensions, e.g., its length, under the influence of gravity (Fig.2).

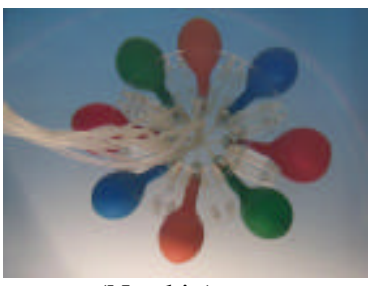

(No skin)

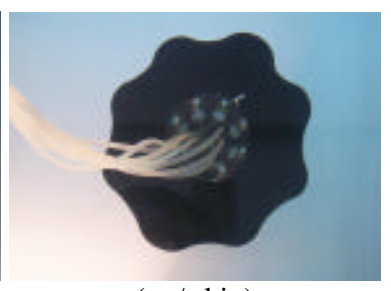

(w / skin)
Figure 1: Amoebot

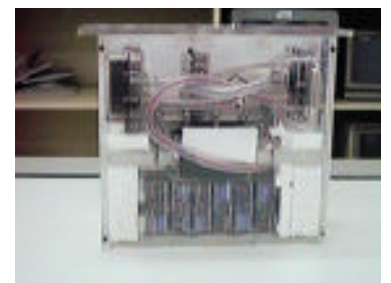

Figure 2: RoboGlider

The study of the two robotic systems complements each other as the current Amoebot can swim in a two-dimensional manner in the water and the RoboGlider travels vertically down to the bottom of the sea floor. The locomotion principles and simulation of both systems based on the theory of the dynamical systems will be introduced. Construction and experiment of those robotic systems under controller environment will be dicussed as well. 


\section{DEVELOPMENT OF AMOEBOT}

\subsection{Locomotion principle}

The unique feature of amoebae's swimming locomotion is the absence of inertia. Fluid dynamics in this situation is characterized by low values of Reynolds number, which is the ratio of the inertia over viscosity. An amoebae squeezes, twists and rotates its body in order to move in a very thick and viscous fluid world. This locomotion has been formulated mathematically and studied in $[1,3,4]$. It is found that the motion is completely determined by the geometry of the sequence of shapes the swimmer assumes in a two-dimensional space. The selfpropulsion comes from slowly changing the body from one shape to another.

\subsection{Mechatronic design and control of $M U V$}

The geometric shape of the real amoebae is a 3D object so that it is capable of generating $3 \mathrm{D}$ motion. To validate the locomotion principle, we use a simplified design so that the shape of the MUV will have planar deformation and the vehicle will produce 2D planar motion. Detailed description of the system is covered in [5].

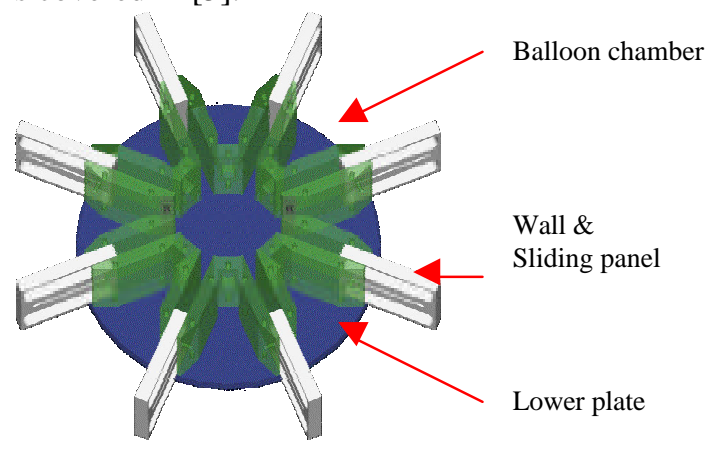

Figure 3: Core structure of MUV

\subsubsection{Core structure}

The core structure of a 2D MUV is a flat hollow cylinder as shown in Fig. 3. The top and bottom faces of the cylinder are made of circular Perspex plates. The diameter and the height of the cylinder are $220 \mathrm{~mm}$ and $90 \mathrm{~mm}$ respectively. Eight open chambers are divided by rigid walls between the two plates and are located along the circumference of the cylinder. The chambers host the inflatable balloons.

\subsubsection{Shape changing mechanism}

The basic consideration in designing the shapechanging mechanism is to maintain minimum shift of the gravity center of the vehicle while the vehicle body deforms. Water-inflated balloons are chosen because they can maintain neutrally buoyant all the time. This design also avoids the waterproof problem since the interior and the exterior of the vehicle are of the same type of the fluid. The balloons are made of latex. The inflation and deflation of balloons are controlled by two-way fluidic valves. Inflated balloons may expand beyond the hosting chamber. The elastic skin wrapped around the entire MUV will then stretch until the tension of the skin is balanced with the internal pressure of the inflated balloons.

\subsubsection{Control system and control sequence}

Because the MUV is designed to create planar motion only, spatial orientation control can be avoided. We mount additional balloons to provide buoyancy perpendicular to the direction of travel to neutralize the gravity. The major concern in controlling this MUV is the geometric shape of the vehicle so that necessary $2 \mathrm{D}$ planar motion can be produced. A hydraulic control system, which consists of a fluidic pump, two-way valves, and a programmable logic controller, is employed. The filling sequences of the balloons are controlled by the PLC. Different sequences can be programmed into the PLC to change the trajectories of the MUV. Because of the space constraint, all the fluidic control circuits are mounted on a fixture outside the vehicle. Right now we would like to investigate how the geometric shape change will affect the movement of the vehicle. Precise control of the shape for steering the vehicle actively is not necessary. An open loop control of the vehicle shape satisfies our objective. The shape control is accomplished through the filling sequences of the balloons programmed into the PLC [5]. Fig. 9 shows the actual and graphical shapechanging stages of one swimming sequence.

\subsection{Experimental Result}

Preliminary experiments have been carried out to establish the performance of the robot. The MUV can stay neutrally buoyant with minimum vertical movement during the experiment. The prescribed shapes derived from the locomotion principle can be produced to high accuracy with the balloon-based shape changing mechanism. The MUV has been allowed to run for many cycles of shape sequence to establish the repeatability of the mechanism. The volume of one fully filled balloon is about 1 liter and it will take about 30 seconds to reach this stage. The initialization sequence takes about 60 seconds. A complete cycle of the swimming sequence takes about 4 minutes. The inflation and deflation rates of the balloons are kept slow to emulate the lowReynolds number world of the amoebae. Irregular self-propulsion has been observed to occur, which at least validates the design principle of a MUV.

\subsection{Swimming rules and trajectory simulation}

The physical size and weight of MUV make the formulation of the swimming rules of amoebae based on vanishing Reynolds number invalid. A new swimming rule has to be established before the trajectory can be predicted. Based on the design of Amoebot, shape produced by inflated balloons can be expressed mathematically in terms of the degree of 
inflation of each balloon. Then, given a sequence of driving shapes and the swimming rule, the trajectory of Amoebot can be simulated and analyzed.

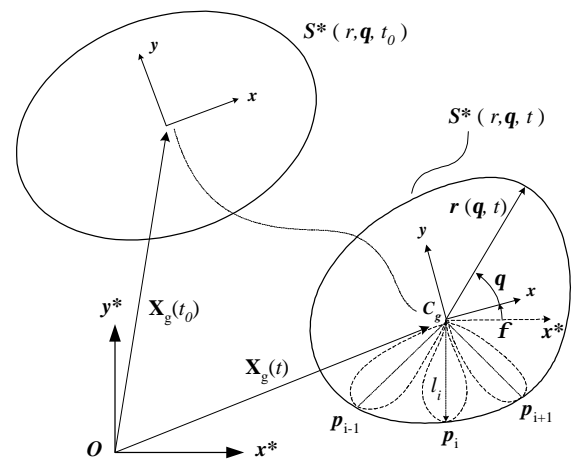

Figure 4: Shape and movement of Amoebot

\subsubsection{Shape of Amoebot}

The shape of the MUV projected on a plane forms an area defined by a closed curve that can be described in a local coordinate frame on the MUV. The origin of the local coordinate frame is chosen at the center of the cylinder, termed the geometric center $\boldsymbol{C}_{g}$ (Fig.4). At any instance $t$, the shape of the robot can be described as $S^{*}(r, \theta, t)$, where $\theta$ is the angle measuring from the $\mathrm{x}$-axis of the body frame. Since the shape is controlled by the inflation of the balloons, we can determine $S^{*}$ as a function of the degree of inflation of the balloons or approximate $S^{*}$ by curve fitting through the farthest points on the balloons. In the current design, the MUV has 8 inflatable balloons providing 8 independent inputs. Let the distance of the farthest point on balloon $i$ to the geometric center $\boldsymbol{C}_{g}$ be $r_{\mathrm{i}}$. All balloons are equally spaced around a circle at 45 ? The 8 necessary conditions to be satisfied for curve fitting are: $r(0, t)=r_{0}, \quad r(\pi / 4, t)=r_{1}, \ldots, r(7 \pi / 4, t)=r_{7}$. Cubic splines are used for the curve fitting of a $C^{2}$ smooth contour curve $r(\theta, t)$ for the body shape. The total area $A$ of the geometric shape is determined by $A(t)=\int_{0}^{2 \pi} \int_{0}^{r(\theta, t)} r^{\prime} d r^{\prime} d \theta$. The surface length $L$, the hydraulic radius $R=A / L$, the moment of inertia $I$, and the center of mass $C_{m}$ are calculated in similar fashion with respect to the shape $S^{*}(r, \theta, t)$ as well.

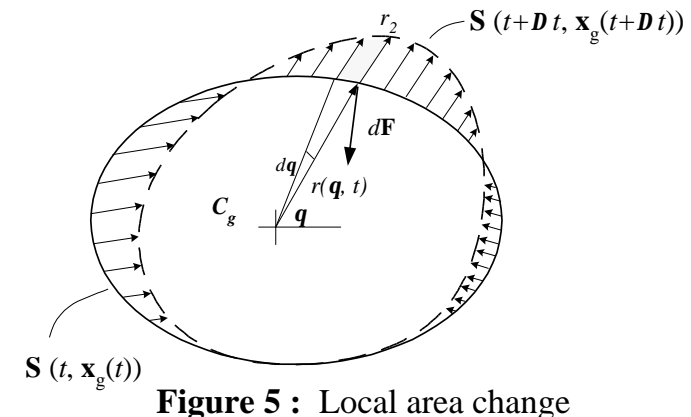

2.4.2. Swimming action

Suppose the geometric center $\boldsymbol{C}_{g}$ of the MUV is located at $\mathbf{x}_{\mathrm{g}}$ with respect to a fixed world reference frame, the shape of the MUV can be described in the world frame through a planar rigid transformation and denoted as $\boldsymbol{S}\left(t, \mathbf{x}_{\mathrm{g}}(t)\right)$. To compare with experimental trajectory and study the dynamics of the MUV swimming, numerical simulation of the swimming based on $\boldsymbol{S}\left(t, \mathbf{x}_{\mathrm{g}}(t)\right)$ can be performed if the swimming rules are known. Classical dynamical theory on the motion of rigid body through infinite inviscid fluid has been formulated by [6]. Following the spirit and formulation based on an impulsive movement, simple robust swimming rules for a real robot in viscous fluid can be derived. Considering a small mass $\Delta m$ within Amoebot, the impulsive change of shape from $\mathbf{S}\left(t, \mathbf{x}_{\mathrm{g}}\right)$ to $\mathbf{S}\left(t+\Delta t, \mathbf{x}_{\mathrm{g}}\right)$ will generate an instantaneous velocity $v$. When Amoebot pushes its surface into the surrounding fluid, a set of rules can be derived based on the surface pressure. For a small surface area $r(\theta, t) d \theta$ (Fig.5), the mass flow rate due to the pushing of the surface from $r(\theta, t)$ to $r(\theta, t+\Delta t)$ can be expressed as $\rho r(\theta, t) d \theta[r(\theta, t+\Delta t)-r(\theta, t)] / \Delta t$. Then, the force acting on the surface when Amoebot pushes the fluid at velocity $[r(\theta, t+\Delta t)-r(\theta, t)] / \Delta t$ can be given as $d \mathrm{~F}$ $=\rho r(\theta, t) d \theta[r(\theta, t+\Delta t)-r(\theta, t)]^{2} / \Delta t^{2}$ acting normal to the surface along the direction of $\vec{\kappa}$. Including only the drag due to the skin friction for dissipation, the resulting linear and angular acceleration when $\Delta t$ goes to zero can be expressed in a coupled differential integral equation as

$$
\begin{aligned}
& \ddot{\mathrm{x}}_{g}(t)=\frac{1}{\gamma A} \int_{0}^{2 \pi} \dot{r}|\dot{r}| r \vec{\kappa} d \theta-\frac{1}{\gamma} \dot{\mathrm{x}}_{g} \\
& \ddot{\theta}(t)=\frac{1}{\gamma I} \int_{0}^{2 \pi} \dot{r}|\dot{r}| r \vec{r} \times \vec{\kappa} d \theta-\frac{2 A R^{2}}{\gamma I} \dot{\theta}
\end{aligned}
$$

Other than neglecting the resultant flow field due to the pushing of the surface, this swimming rule actually models the swimming mechanism of reallife animals, which paddles fluid to the back in order to swim forward. Due to the nature of the swimming mechanism, it can be called the Momentum Rule. Another swimming rule based on the microorganism swimming was formulated, termed the Energy rule, and introduced in [8].

\subsubsection{Simulated trajectory}

Trajectories based on the above rule have been obtained by integrating the translation and rotation equations in time from a set of initial conditions. The swimming is driven by having Amoebot to squeeze its mass suddenly towards the desired direction of travel and relax back to its original distribution slowly. The simulated trajectory of Amoebot based on the Momentum rule is shown in Fig.6. The set of functions drives Amoebot towards a direction between the north and northwest. The shapes of Amoebot return to its initial geometry after $t$ goes from 0 to 1 . A new cycle will start when $t>1$. Details of the driving conditions are described in [8]. 
Within the same time period $[0,1]$ under the same driving sequence, $\mathrm{C}_{\mathrm{g}}$ moves from $(0,0)$ to $(0.0705$, 0.1680 ), a net traveling distance of 0.09 body length approximately. The body maintains its orientation, i.e., $\theta \approx 0^{\circ}$. Because the robot stretches in the northwest direction and retracts in other directions symmetrically, the reaction force exerted by the surrounding water will make the robot move in the direction opposite to the one produced by Energy rule. The backward acceleration due to the slow retracting is insignificant compared with the net velocity at the end of the strong forward motion.

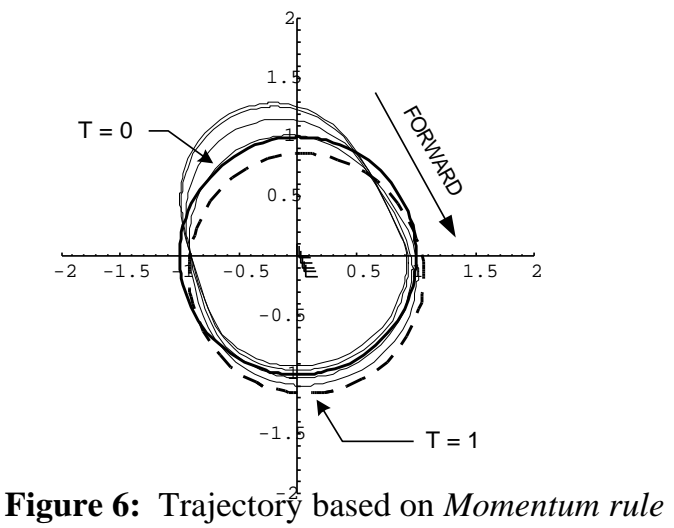

\section{DEVELOPMENT OF ROBOGLIDER}

\subsection{Dynamics of falling paper}

The design of RoboGlider was inspired by the falling leaf phenomena. The falling motions of a paper is complex and rich in hydrodynamic effects, including lift, drag, vortex shedding and stall. There have been previous mathematical models describing the motion of a falling light, thin object $[6,9,10]$. However, a complete solution for the problem and correct descriptions for the motions remains undetermined. Tanabe and Kaneko [2] put another approach to the study of motion of falling a thin object. In their model, the paper is treated as a rigid body with zero thickness, and only its length and weight characterize the body. The paper is subjected to three types of forces: gravity, lift and friction. Through the experiment, five falling motions were demonstrated. The five falling motions are periodic rotation/fluttering, chaotic rotation/fluttering and simple perpendicular fall. These motions were successively found by changing the drag coefficient of the object in the air. Belmonte, Eisenberg and Moses [11] conducted a similar experiment on a flat strip and observe two fundamental motions: side to side oscillation (flutter) and end-over-end rotation (tumble). The motions are shown in Fig.7. They found that the transition from flutter to tumble is determined by the Froude number, Fr, at high Reynolds number environment. The Froude number is the ratio of characteristic times for downward motion and pendulum oscillations, defined as
$F r=\sqrt{\frac{M}{\rho L^{2} w}}$, where $M$ is the mass, $\rho$ is the density, $L$ is the length, and $w$ is the width of the strip. From the experiment, it showed that the friction effect is mainly the inertia drag, the effect of added mass is insignificant.

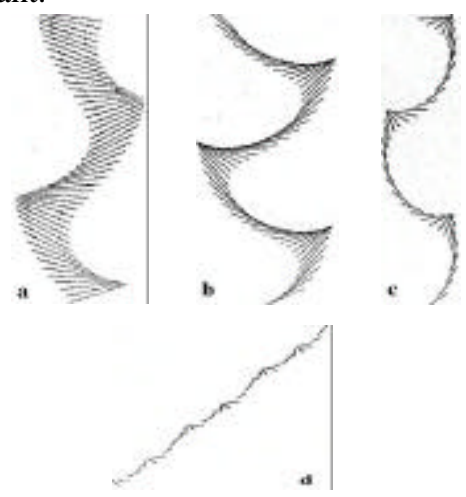

Figure 7: Trajectories of a falling stripwith different Froude Numbers [7]

\subsection{Modeling of RoboGlider}

The idea of RoboGlider grew out of a strip-like underwater autonomous system that can change its length - the characteristic dimension. Using a retractable strip platform, the length of the autonomous mechanical system can be changeable. As indicated in the falling paper effect, changing the length of the strip can produce different falling motions. With the length of the strip controllable, the trajactory and orientation of the system could be controlled during the undewater deployment. Based on the simple model of [2] and the subsequent experimental result of [11] we develop the dynamic model of a strip type underwater system with retractable platform that will fall under the gravity. For simplicity, we restrict this system moving in 2D space. As shown in Fig.8, let $(\mathrm{x}, \mathrm{y})$ be the position of the platform, $V=\left(v_{x}, v_{y}\right) \equiv\left(\frac{d x}{d t}, \frac{d y}{d t}\right)$ is the system velocity. The friction experienced by the system can be described by two perpendicular components, $F_{\perp}=-m_{p} k_{\perp}\left(v_{y} \cos \theta-v_{x} \sin \theta\right)$ and

$F_{=}=-m_{p} k_{=}\left(v_{y} \sin \theta+v_{x} \cos \theta\right)$, where $m_{p}$ is the mass of the system, $k_{\perp}$ and $k_{=}$are friction coefficents in the directions perpendicular and parallel to the platform respectively. The friction due to the rotation of the platform can be expressed as $F_{\theta}=-m_{p} k_{\text {perp }} l^{2} \omega / 12$. Assume that the fluid is imcompressible and stationary. According to KuttaJoukowski Theorem, the direction of the lift force is against the falling direction of the research object. Hence, when studying the lift, the velocity of the moving platform and should be seperated from the overall system as the platform has its own velocity. Let the velocity of the platform be $V_{\text {plat }}$ and the angle between the velocity and the vertical direction be $\beta$. The displacement of the moving platform is denoted 
$\mathrm{u}(\mathrm{t})$. We have $V_{\text {plat }}^{2}=V^{2}+\dot{u}^{2}(t)-2 V \dot{u}(t) \sin (\theta+\alpha)$, $\beta=\cos ^{-1}\left(\frac{V-\dot{u}(t) \sin (\theta+\alpha)}{\sqrt{V^{2}+\dot{u}^{2}(t)-2 V \dot{u}(i) \sin (\theta+\alpha)}}\right)-\alpha$.

The total lift on the system is thus,

$L_{x}=\gamma \rho_{f} \pi\left[l V^{2} \cos (\alpha+\theta) \cos \alpha+\kappa u(t) V_{\text {plat }}^{2} \cos (\beta-\theta) \cos \beta\right]$

$L y=-\gamma \rho_{f} \pi\left[l V^{2} \cos (\alpha+\theta) \sin \alpha-\kappa u(t) V_{\text {plat }}^{2} \cos (\beta-\theta) \sin \beta\right]$

where

$\gamma=\left\{\begin{array}{cc}1, & v_{y}<0,0<\alpha+\theta<\pi, \text { or } v_{y}>0,-\pi<\alpha+\theta<0 \\ -1, & v_{y}<0,-\pi<\alpha+\theta<0, \text { or } v_{y}>0,0<\alpha+\theta<\pi\end{array}\right.$

$\kappa=\left\{\begin{array}{cc}-1, & V_{y, p l a t}<0,0<\beta-\theta<\pi \text { or } V_{y, \text { plat }}>0,-\pi<\beta-\theta<0 \\ 1, & V_{y, p l a t}<0,-\pi<\beta-\theta<0 \text { or } V_{y, p l a t}>0,0<\beta-\theta<\pi\end{array}\right.$

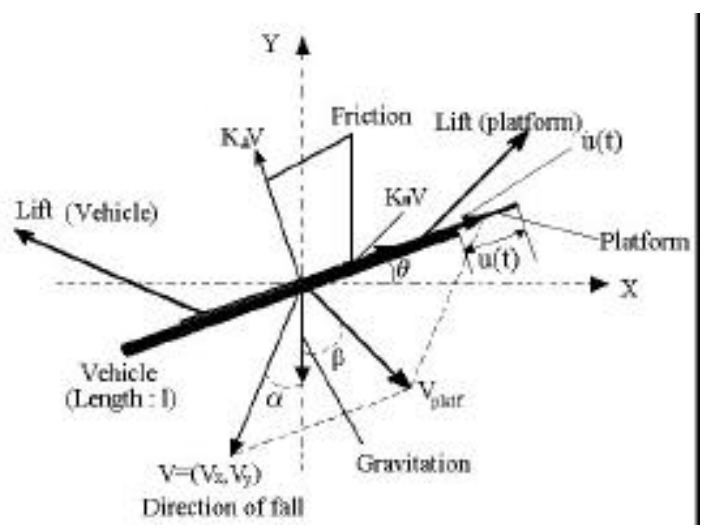

Figure 8: Model of RoboGlider

Considering the moment produced by the retractable platform and the rotaional inertia of the system, we can obtain the following dynamical model to describe the movement of the system:

$\frac{d x}{d t}=v_{x}, \frac{d y}{d t}=v_{y}, \frac{d \theta}{d t}=\omega$

$\frac{d v_{x}}{d t}=-\left(k_{\perp} \sin ^{2} \theta+k_{=} \cos ^{2} \theta\right) v_{x}+\left(k_{\perp}-k_{=}\right) \sin \theta \cos \theta v_{y}$

$+\gamma \frac{\rho_{f} \pi}{m_{p}}\left[V_{\text {plat }}^{2} l \cos (\alpha+\theta) \cos \alpha+\kappa V_{\text {plat }}^{2} u(t) \cos (\beta-\theta) \cos \beta\right]$

$\frac{d v_{y}}{d t}=-\left(k_{\perp} \cos ^{2} \theta+k_{=} \sin ^{2} \theta\right) v_{y}+\left(k_{\perp}-k_{=}\right) \sin \theta \cos \theta v_{x}$

$-\gamma \frac{\rho_{f} \pi}{m_{p}}\left[V_{\text {plat }}^{2} l \cos (\alpha+\theta) \sin \alpha-\kappa V_{\text {plat }}^{2} u(t) \cos (\beta-\theta) \sin \beta\right]-g$

$\frac{d \omega}{d t}=-k_{\perp} \omega-\frac{3 \rho_{f} \pi}{m_{p} l^{2}}\left[V_{p}^{2} l^{2} \cos (\alpha+\theta) \sin (\alpha+\theta)\right.$

$\left.+u(t)^{2} V_{\text {plat }}^{2} \cos (\beta-\theta) \sin (\beta-\theta)\right]$

Trajectory simulation of the system under sinusoidal input $u(t)=\varepsilon \sin \omega t$ is shown in Fig.10. As discussed in [2,11], the falling of paper or the platform sometimes exhibits periodic motions. Here is our first attempt to see how the dynamic system will respond to the sinusoidal change of its characteristic length. The parameters of the system [2] are: $\mathrm{k}_{\text {perp }}=10.0, \mathrm{k}_{\text {para }}=1000, \rho=0.1, l=1.0 \mathrm{~m}$. The initial conditions of 5 cases are the same. The total simulation time is $30 \mathrm{sec}$. When the frequency of the length change is close to or smaller than the period of fallig motion $(\omega=0.4 \pi)$ (Fig.10 A,D), the platform can be controlled to fall vertically. When increases or the amplitude of the change, the periodic falling motion disappear (Fig.10 B,C,E). Further study on its dynamic effect is required.

\subsection{Mechatronic design of RoboGlider}

To validate the concept of the retractable platform robotic system, we designed a prototype RoboGlider, a thin-box like shape with a one-DOF movable platform on top (Fig.2). The robot measures $180 \mathrm{~mm} \times 180 \mathrm{~mm} \times 66 \mathrm{~mm}$. The robot is restricted to moving in a slab-shaped watertank with $2 \mathrm{D}$ motions$(\mathrm{x}, \mathrm{y}, \theta)$. Although conceptually the falling leaf and the retractable platform model of Section 3.2 are basically 1D objects, the 2D RoboGlider will exhibit similar behavior because the aspect ratio is actually factored in the study of [2] and [11].

The construction of the RoboGlider uses perspex and a radio-controlled servo motor. The sliding motion of the top platform is actuated by a servo motor through a 4-bar linkage. In dealing with the waterproof problem of the overall system, we designed a set of gears - waterproofed servo motors, controllers, and radio receivers, to facilitate the rapid development of underwater mechanical systems. Being water-tight for individual components, there is no need to seal off the entire robot. In addition, maintenance of the system will be much easier than the sealed-off systems. For the control system, a BasicStamp microcontroller is used to regulate the DC servo motor. A radio transmitter unit is mounted on the robot to receive command from the remote $\mathrm{RC}$ controller. With this set up, we can easily debug the entire system. A computer-controlled vision system is currently being set up for the visual-servoing the robot motion in autonomous mode.

\subsection{Preliminary experiment}

Using the RC remote controller, we are able to do some preliminary testing. The experiment shot are shown in Fig.11. The top platform extruded out to the left $40 \mathrm{~mm}$ along the vertical drop test. And the robot actually falled to the right about $130 \mathrm{~mm}$ to the centerline marked on the watertank.

\section{CONCLUSION}

We presented here two types of underwater deployment robot systems without propellers: Amoebot and RoboGlider. The propulsions of those systems is based on biological systems, which are effective and environment friendly. Theoretical investigations and simulations of the propulsion methods are presented. Prototypes of those systems are constructed and are currently under extensive tests. Preliminary result of the experiments show that those novel propulsion methods are feasible. 
Experiments on controlled motions of the Amoebot and RoboGlider will be conducted in the near future to validate the proposed control algorithms.

Acknowledgements: This research is supported by Ministry of Education, Singapore under Academic Research Project RG68/98.

\section{REFERENCES}

[1] A. Shapere, F. Wilczek, Geometry of self-propulsion at low Reynolds number, J. Fluid Mech., V.198, pp 557-585, 1989.

[2] Y. Tanabe, K. Kaneko, Behavior of a Falling Paper, Phys. Review Letters, V.73, N.10, pp1372-1375, 1994.

[3] A. Shapere, F. Wilczek, Efficiencies of self-propulsion at low Reynolds number, J. Fluid Mech., V.198 pp 587$599,1989$.

[4] T.J. Pedley, J.O. Kessler, Hydrodynamic phenomena in suspension of swimming microorganism, Annu. Rev. Fluid Mech., V.24, pp313-358, 1992.

[5] A. Cathala, Marine robot propelled by microorganism locomotion principle: Amoebot, Technical report, School of Mechanical and Production Enigneering, Nanyang Technological University, 1998.

[6] H. Lamb, Hydrodynamics, Dover Pub., USA, 1945.

[7] H.-S. Li, I.-M. Chen, Swimming Dynamics of a ShapeChanging Object, submitted to ASME J. Dyn Sys, Measurement, and Control.

[8] I.-M. Chen, H.-S. Li,, A. Cathala, Design and Simulation of Amoebot, A Metamorphic Underwater Vehicle, IEEE Int. Conf. Robotics Auto., Detroit, USA, 1999, pp90-95.

[9] J. C. Maxwell. The Scientific Papers of James Clerk Maxwell, pp115-118, Dover Pub., USA, 1890.

[10] H. J, Lugt. Annu. Rev. Fluid Mech. V.15, N. 123, 1983.

[11] A. Belmonte, H. Eisenberg, E. Moses, From Flutter to Tumble: Inertial Drag and Froude Similarity in Falling Paper, Phys. Review Letters, V.81, N.2, pp345-348, 1998.

[12] Y.H. Poh, Development of An Underwater Deployment Robotic Platform, FYP Report, School of Mechanical and Production Engineering, Nanyang Technological University, 2000.
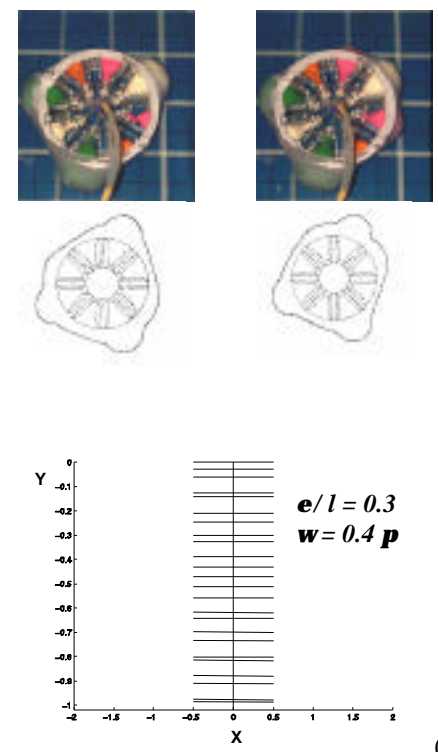

(A)
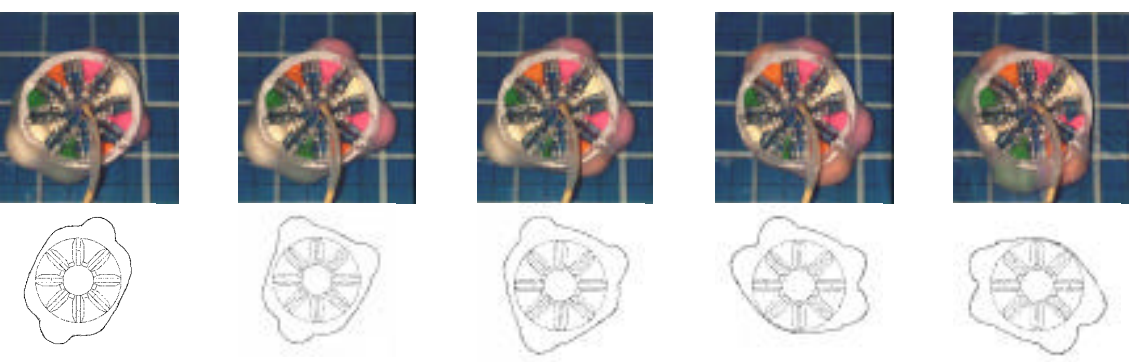

Figure 9: Swimming sequence of Amoebot

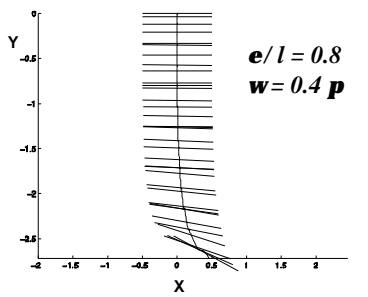

(B)

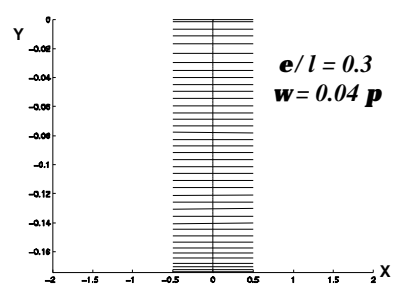

(D)

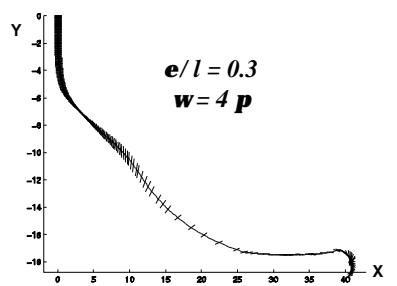

(E)
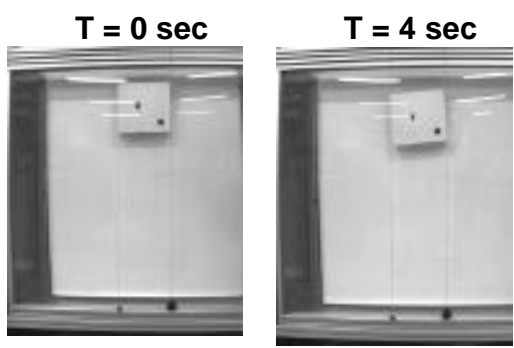

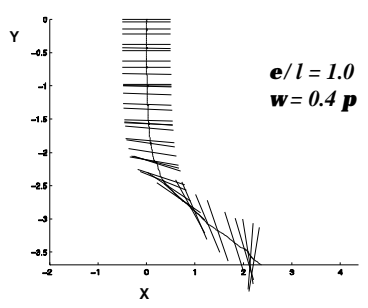

(C)

Figure 10:

Trajectory simulation of RoboGlider.

Initial condition $(x, y, \theta)=(0,0,0)$

Total time $=30 \mathrm{sec}$
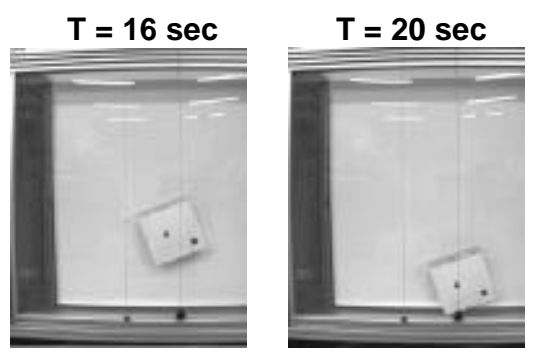

Figure 11: Experiment of RoboGlider 